This manuscript was accepted by J. Appl. Phys. Click here to see the version of record.

\title{
Photonic nanojets with mesoscale high-index dielectric particles
}

\author{
V. Pacheco-Peña, ${ }^{1, a}$ and M. Beruete,,$^{2,3, b^{*}}$
}

${ }^{1}$ School of Engineering, Newcastle University, Merz Court, Newcastle Upon Tyne, NE1 7RU, UK.

${ }^{2}$ Antennas Group-TERALAB, Universidad Pública de Navarra, Campus Arrosadía, 31006 Pamplona, Spain

${ }^{3}$ Institute of Smart Cities (ISC), Public University of Navarra, 31006 Pamplona, Spain

In this work, we demonstrate the ability of high-index dielectric particles immersed in air to generate photonic nanojets with extreme resolution $\left(\sim 0.06 \lambda_{0}\right)$. Both 2D (cylindrical) and 3D (spherical) particles are analyzed and their profile is truncated using the Weierstrass formulation for solid immersion lenses to produce a photonic nanojet at the output surface under planewaye illumination. Their focusing capability is evaluated in terms of the spatial resolution achieving subwavelength values of $\sim 0.14 \lambda_{0}$ and $\sim 0.06 \lambda_{0}$, for a truncated cylinder and sphere respectively. The capability of the truncated sphere to enhance the backscattering produced by two small metallic spherical scatterers placed near the photonic nanojet is evaluated by using a scanning-probe microscopy configuration. The imaging capabilities of this technique are also analyzed by moving the metallic spheres in the transversal plane where the photonic nanojet is produced. The results here presented improve greatly the typical resolution of photonic nanojets generated with dielectric particles with small index contrast. In addition, the highindex material allows using mesoscale particles, leading to a more compact setup. These results may find applications in areas such as microscopy, imaging and sensing devices where a subwavelength resolution below the diffraction limit is needed.

\footnotetext{
a) Electronic mail: victor.pacheco-pena@newcastle.ac.uk

b) Electronic mail: miguel.beruete@unavarra.es
} 


\section{Publishing I. INTRODUCTION}

As it is known, the spatial resolution of an imaging system is naturally limited by the diffraction of electromagnetic waves because the scattered field produced by small features is mainly evanescent, hindering their detection ${ }^{1}$. The necessity to improve the performance of imaging systems for microscopy applications resolving the subwavelength features of an object has pushed forward the scientific community in order to overcome this diffraction limit. Different methods and techniques have been proposed such as solid immersion lenses $^{2-4}$, diffractive optics ${ }^{5}$, microspherical dielectrics ${ }^{6-8}$, superoscillatory devices $^{9-11}$ and metamaterials ${ }^{12-15}$. In this realm, photonic nanojets (PNJs) were first proposed and demonstrated several years ago at optical frequencies by using micrometer scaled dielectric elements with cylindrical (2D) and also spherical (3D) geometries $^{16-21}$.

PNJs are high intensity, non-evanescent and narrow beams generated just at the output surface of dielectric particles, usually cylinders or spheres, when they are illuminated with a planewave. The typical approach to generate PNJs is to fix a relatively small index contrast between the dielectric particle and the background medium, usually less than two $\left(n / n_{0}<2\right)^{18,22}$ but also with an index contrast close to unity ${ }^{23}$ when working in reflection mode. With this strategy a subwavelength spatial resolution of $\lambda_{0} / 3$ (where $\lambda_{0}$ is the operational wavelength) has been obtained with PNJs generated by dielectric microspheres, beating the diffraction limit ${ }^{24,25}$. One limitation of this approach is that the particles must have a large size (around five wavelengths) in order to achieve subwavelength resolution. In addition to spheres and cylinders, alternative geometries to produce PNJs have been suggested such as core-shell dielectrics ${ }^{26,27}$, hemispherical and elliptical particles ${ }^{28,29}$, dielectric cuboids $^{30-32}$ and graded index structures ${ }^{33}$. They have been proposed and demonstrated in different areas such as waveguiding ${ }^{34-36}$, directional emission ${ }^{37}$, surface plasmons ${ }^{38,39}$, optical trapping ${ }^{40,41}$ backscattering $^{16,21,42,43}$, Raman signal enhancement ${ }^{44,45}$ and also working in reflection mode ${ }^{44,46}$, demonstrating to be good candidates to overcome the diffraction limit in different fields and spectral ranges.

To push forward the limits and implement PNJs with extreme subwavelength spatial resolution, in this manuscript we propose a provoking concept that consists basically in using a large index contrast between the dielectric particle and the background medium, $n / n_{0} \gg>2$, breaking the typical condition of mild index contrast,

$n / n_{0}<2$. This way, we can boost the resolution and, at the same time, reduce the particle to a mesoscale size (comparable to the operation wavelength). Both cylinders and spheres are evaluated and to achieve PNJs with subwavelength resolution their profile is shaped using the Weierstrass formulation for immersion lenses ${ }^{2}$. The spectral response of the generated PNJs is studied in detail and the backscattering enhancement and imaging 


\section{MESOSCALE HIGH-INDEX CYLINDER (2D)}
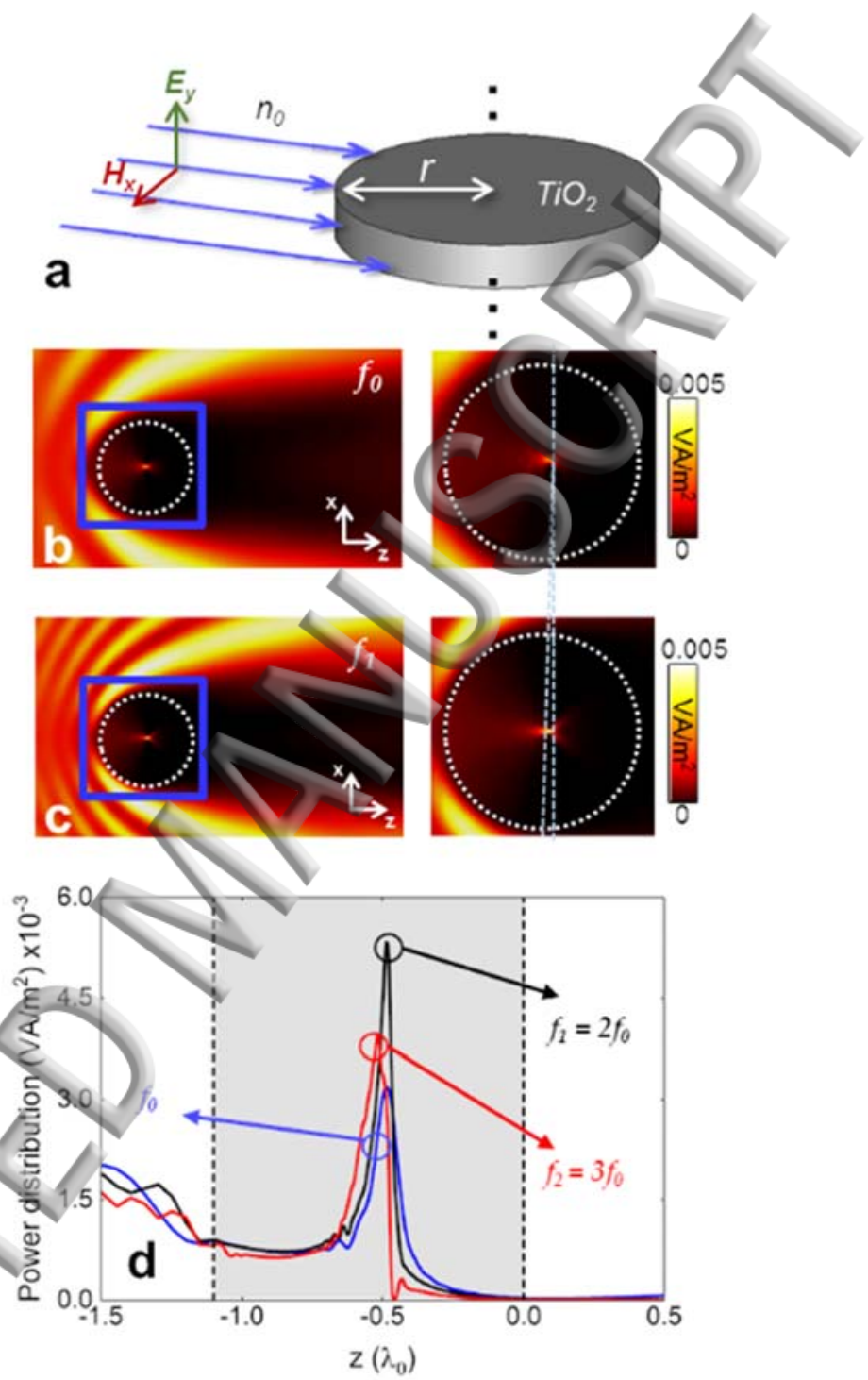

FIG.1 (a) Schematic representation of a high-index cylinder with radius $r=0.55 \lambda_{0}$ immersed in air under planewave illumination with vertical polarization, $E_{y}$. Numerical results of the power distribution on the $x-z$ plane at (b) $f=f_{0}$ and (c) $f=$ $f_{l}=2 f_{0}$. The right panels in (b,c) correspond to a zoom-in picture of the area delimited in the left panels with a blue square. In the same panels, the vertical white dashed lines represent the displacement of the PNJ when the operation frequency is changed from $f_{0}$ to $f_{l}$. As observed, one of these lines is tilted showing how the PNJ is moved inside of the high-index cylinder. To guide the eye, the cylinder is represented with white dotted lines in (b,c). (d) Power distribution along the $z$-axis at three different harmonics $f_{0}$ (blue), $f_{1}=2 f_{0}$ (blue) and $f_{2}=3 f_{0}$ (red).

All the numerical simulations in this manuscript are carried out using the transient solver of the commercial software CST Microwave Studio ${ }^{\circledR}$. In the following discussion the spectral response of the PNJs is evaluated at the fundamental design frequency $\left(f_{0}=50 \mathrm{GHz}\right)$ and integer harmonics of it, $f_{m}=(m+1) f_{0}$ with $m=0,1,2$. 


\section{This manuscript was accepted by J. Appl. Phys. Click here to see the version of record.}

Titanium Dioxide $\left(\mathrm{TiO}_{2}\right)$ is used for the dielectric particles since it has a high permittivity, $\varepsilon_{r} \sim 92+i 4.6(\operatorname{Re}\{n\}$

$\sim 9.59)$ and relatively low loss at microwave frequencies $(\tan \delta \sim 0.05)^{47}$ and the particles are in all cases embedded in air $\left(n_{0}=1\right)$.

In the first study, we consider a high-index cylinder with radius of $0.55 \lambda_{0}$, where $\lambda_{0}=6 \mathrm{~mm}$ is the wavelength at the design frequency, see scheme in Fig. 1a. It is illuminated with a vertically polarized planewave $\left(E_{y}\right)$ propagating along the $z$ axis (as shown in the same figure). In the simulation, top and bottom electric plane boundary conditions are used to emulate an infinite cylinder along the $y$ axis. With this setup, the numerical results of the power distribution on the $x-z$ plane at $f_{0}$ shown in Fig. $1 \mathrm{~b}$ are obtained. A clear high intensity spot is noticed inside the cylinder, unlike classical PNJs that appear on the surface of the dielectric particles. To better appreciate it, a zoom-in image is shown on the right-side panel of the same figure. This is an expected result because of the high refractive index contrast between $\mathrm{TiO}_{2}$ and $\mathrm{air}\left(n / n_{0} \sim 9.59\right)^{18}$ that deviates from the condition $n / n_{0}<2$ to have a PNJ on the surface ${ }^{18,22}$. As shown in Fig. $1 \mathrm{c}$, at $f_{l}=2 f_{0}$ the PNJ is further shifted to the left inside the cylinder. For completeness, the numerical results of the power distribution along the $z$-axis at $f_{0}, f_{l}$ and $f_{2}$ are shown in Fig. 1d, where it is clear that the focus is always inside the cylinder in all cases.

Based on the results shown in Fig. 1, to shift the position of the PNJ towards the surface one may think of different options: (i) to immerse the cylinder inside a higher-index medium reducing the refractive index contrast $n / n_{0}{ }^{18}$; or (ii) to change the geometry of the cylinder. Since our aim here is to work with a high refractive index contrast, the shape of the cylinder will be engineered to generate a PNJ just at the border. A way to do this would be by parametrically optimizing the geometry of the high-index cylinder via numerical simulations ${ }^{29}$. However, an analytical approach to get the optimal shape is preferable for design purposes. Therefore, here the geometry is obtained by exploiting the Weierstrass formulation for solid immersion lenses ${ }^{2}$. The schematic representations of the initial and truncated cylinder are shown in Fig. 2a. The parameter $a$ (which is the distance between the center of the cylinder to the edge where the profile is truncated to a flat surface) can be calculated as:

$$
a=[1+1 / n] r-r
$$

where $n$ is the refractive index of the cylinder. With this setup, and considering a cylinder with $r=0.55 \lambda_{0}$, the truncation is applied at a distance $a=0.057 \lambda_{0}$ from the center. 

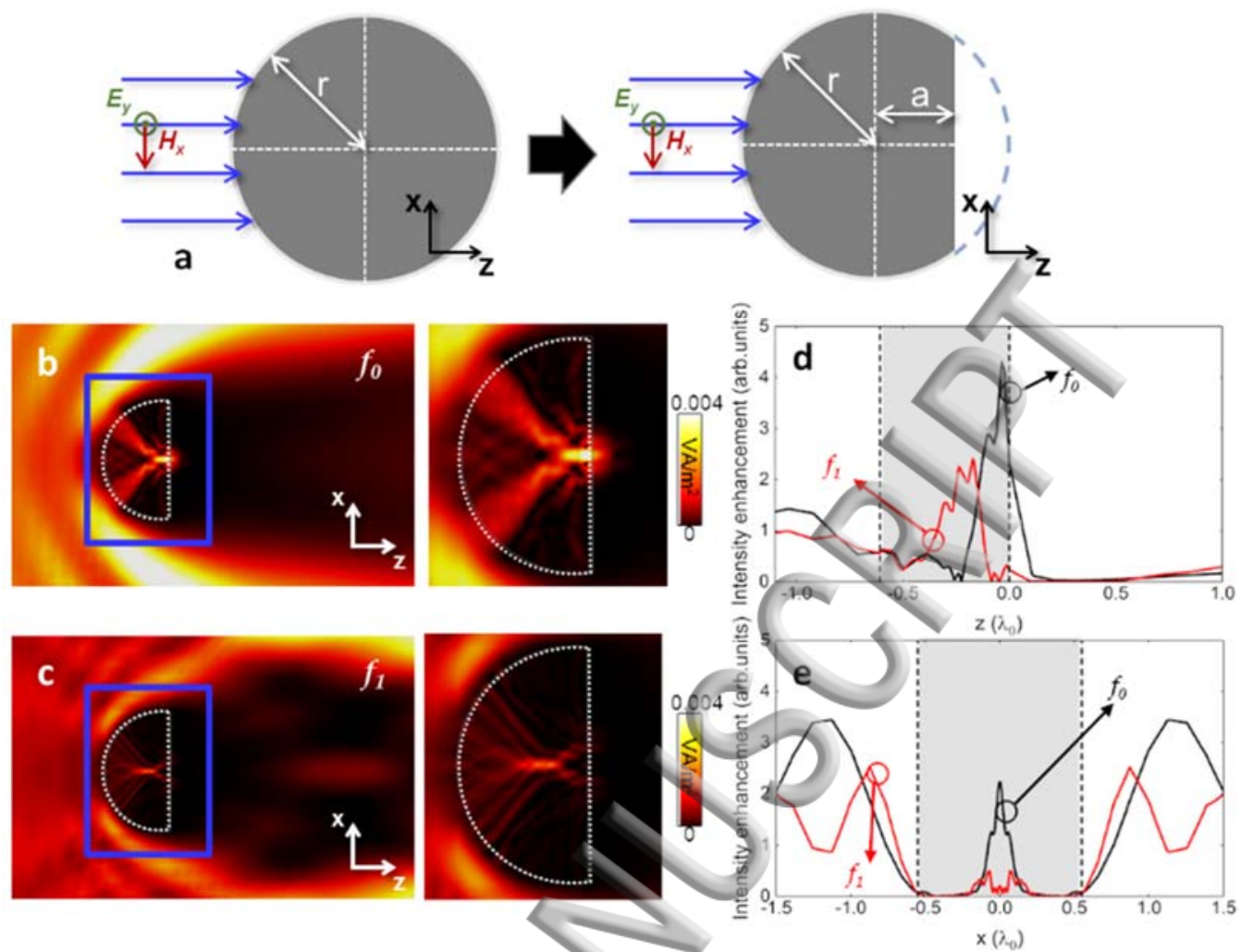

FIG.2 (a) Schematic representation of a high-index regular (left) and truncated cylinder with $r=0.55 \lambda_{0}$ immersed in air under planewave illumination with vertical polarization, $E_{y}$. Numerical results of the power distribution on the $x-z$ plane for the truncated cylinder at (b) $f=f_{0}$ and (c) $f=f_{1}=2 f_{0}$. The right panels in (b,c) correspond to a zoom-in picture of the area shown in the left panels delimited with a blue square. To guide the eye, the truncated cylinder is represented as the white dotted lines in $(\mathrm{b}, \mathrm{c})$. Intensity enhancement of the truncated cylinder at $f_{0}$ (black) and $f_{l}=2 f_{0}$ (red) along the (d) propagation $z$-axis and (e) transversal $x$-axis at $z=0$.

The truncated cylinder response is numerically evaluated following the same setup as in Fig. 1 and the results of the power distribution on the $x-z$ plane at $f_{0}$ and $f_{1}$ are shown in Fig. 2b,c, respectively (a zoom-in image of the power distribution is also shown on the right-hand side of each panel for completeness). A clear PNJ is obtained at the output surface only at $f_{0}$ (Fig. 2b), whereas the high intensity spot is shifted inside the cylinder at $f_{l}$ (Fig. 2c). To better compare the performance, the numerical results of the intensity enhancement (calculated as the ratio of the power distribution with and without the truncated cylinder) along the $z$-axis (at $x=y=0$ ) is shown in Fig. 2d. An intensity enhancement of 2.5 is obtained near $z=0$ at the fundamental frequency $f_{0}$. Additionally, the intensity enhancement along the transversal $x$-axis (at $y=z=0$ ) is plotted in Fig. 2e. A clear focus is obtained at $f_{0}$ with a Full-Width at Half-Maximum (defined as the distance at which the intensity enhancement has decayed half its maximum along the transversal axis) $F W H M_{x}=0.14 \lambda_{0}$ demonstrating the ability to produce a PNJ with a high spatial resolution. In contrast, no PNJ is produced at the output surface at $f_{l}$ due to the fact that the focus is inside (Fig. 2c). 


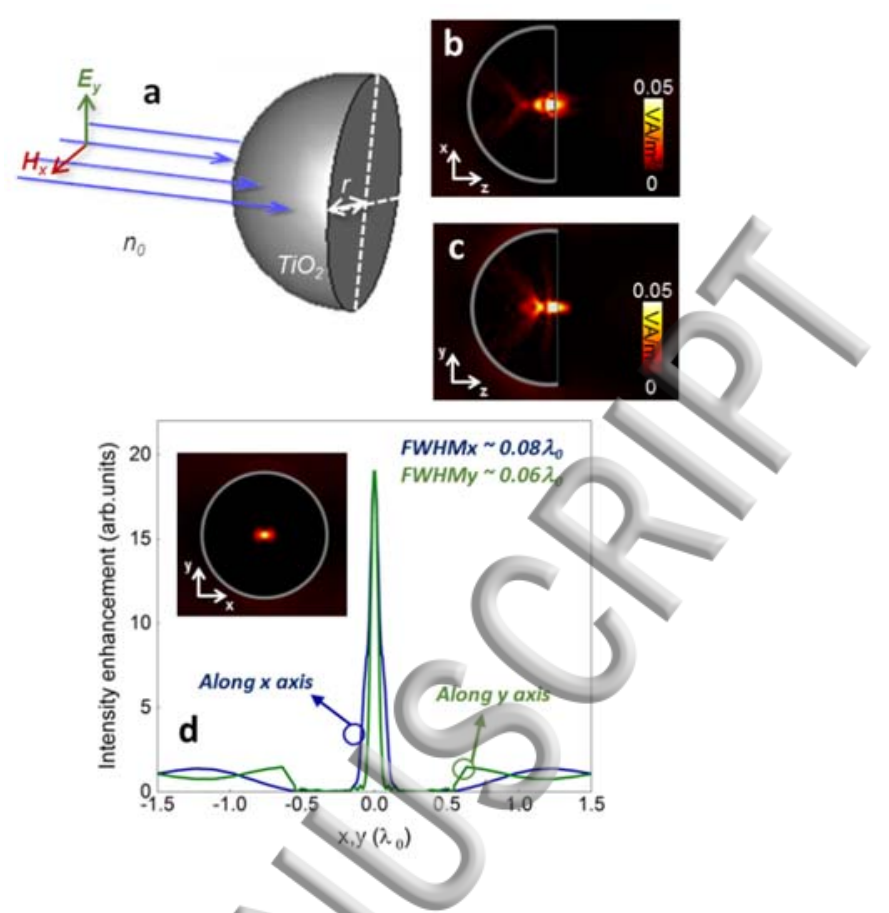

FIG.3 (a) Schematic representation of a truncated high-index sphere with $r=0.55 \lambda_{0}$ immersed in air under planewave illumination with vertical polarization, $E_{y}$. Numerical results of the power distribution on the (b) $x-z$ plane and (c) $y-z$ plane. (d) Intensity enhancement along the transversal $x$-(dark blue) and $y$ - (green) axis at $y=z=0$ and $x=z=0$, respectively. The power distribution on the $x-y$ plane at $z=0$ is shown as inset in panel (d) for completeness.

In the previous section, the ability to generate PNJs with a high-index truncated cylinder was evaluated. From the experimental point of view, it is also important to evaluate the performance of 3D particles. To do this, we consider the truncated dielectric sphere shown in Fig. 3a. As in the previous section, $\mathrm{TiO}_{2}$ and air are chosen as the high dielectric material and background medium, respectively. The profile of the truncated sphere is calculated using Eq. 1 and applying rotational symmetry to the truncated dielectric cylinder shown in Fig. $2 b$. For the numerical evaluation, open expanded boundary conditions are used in all directions $(x, y, z)$ to embed the sphere in air. The excitation is done with a vertically polarized planewave is used $\left(E_{y}\right)$, and to reduce the computational effort electric and magnetic symmetries are applied on the $x-z$ and $y-z$ planes, respectively.

With this setup, the numerical results of the power distribution at $f_{0}$ along the $H$-plane $(x-z)$ and $E$-plane $(y$ z) are shown in Fig. 3a,b, respectively. As observed, a clear PNJ is obtained at the output surface of the truncated sphere. To better compare these results, the numerical results of the intensity enhancement along the $x$-axis (at $y=z=0$ ) and $y$-axis (at $x=z=0$ ) are shown in Fig. 3d. A high spatial resolution is achieved with values of $F W H M_{x}=0.08 \lambda_{0}$ and $F W H M_{y}=0.06 \lambda_{0}$ along the $x$ and $y$ axes, respectively, and an intensity enhancement at the focal point of $\sim 18$. 


\section{This manuscript was accepted by J. Appl. Phys. Click here to see the version of record.}

\section{BACKSCATTERING ENHANCEMENT}

To evaluate the backscattering enhancement and imaging capabilities of the truncated sphere, small metallic spherical scatterers are introduced within the PNJ produced at the output. The backscattering enhancement is then calculated as in ${ }^{30}$ as the ratio between Radar Cross Section (RCS) at the backward direction produced with and without scatterers at the output of the truncated sphere. Note that this configuration can be considered as a variation of scanning-probe microscopy technique ${ }^{18,48}$ where the probe corresponds to the PNJ produced by the high-index dielectric instead of a needle-like structure ${ }^{49}$. The calculations are performed following the setup shown in Fig. 4a,f. The scatterers are two subwavelength metallic spheres made of gold (Au) with nominal conductivity $\sigma_{\mathrm{Au}}=4.561 \times 10^{7} \mathrm{~S} / \mathrm{m}$ and a diameter of $0.067 \lambda_{0}$ separated by a distance $\Delta x$ and $\Delta y$ when they are aligned along the $x$ or $y$ axis, respectively (see Fig. 4a and Fig. 4f for each case, respectively). They are placed at the output surface of the truncated sphere and are shifted along the $x$ or $y$ axis (depending on the axis where the spheres are lying) from $-0.267 \lambda_{0}$ to $0.267 \lambda_{0}$ with a step of $0.0067 \lambda_{0}$. It is important to note that the same results would be obtained if the metallic scatterers were spatially fixed and the high-index dielectric sphere were moved along the $x$ or $y$ transversal directions. In this manuscript however, the scatterers are moved keeping the high-index sphere in a fixed position, for simplicity in the calculation (to avoid changing the air-box used as background medium. In this way, all the simulations are calculated using the same configuration). For a complete evaluation of the performance, at each position, the distance between them (either $\Delta x$ or $\Delta y$ ) is varied from $0.013 \lambda_{0}$ to $0.13 \lambda_{0}$ with a step of $0.013 \lambda_{0}$.

With this setup, the numerical results of the backscattering enhancement maps obtained when $\Delta x$ or $\Delta y$ is varied are shown in Fig. 4b,g, as a function of the position along the $x$ or $y$ axis, respectively. A strong backscattering enhancement appears when the scatterers are near the PNJ and their positions along the $x$ or $y$ axis are clearly observed (white regions in Fig. 4b,g, respectively). To better compare these results, the backscattering enhancement using different values of $\Delta x$ and $\Delta y\left(0.13 \lambda_{0}, 0.06 \lambda_{0}\right.$ and $\left.0.013 \lambda_{0}\right)$ are extracted from Fig. 4b,g and are depicted in Fig. 4c-e and Fig. 4h-j, respectively.

From the results shown in Fig. 4c-e, it is inferred that for $\Delta x=0.013 \lambda_{0}$ (Fig. 4e) the spatial resolution of the

PNJ is not sufficient to detect this small distance between the metallic spheres. However, the truncated sphere is able to detect subwavelength features as small as $0.06 \lambda_{0}$ (and larger), as observed in Fig. 4c-d. Similar performance is achieved when the metallic spheres are placed along the $y$ axis and $\Delta y$ is modified (see Fig. 4hj) demonstrating the backscattering enhancement capabilities of the proposed PNJ produced by the truncated high-index sphere. 

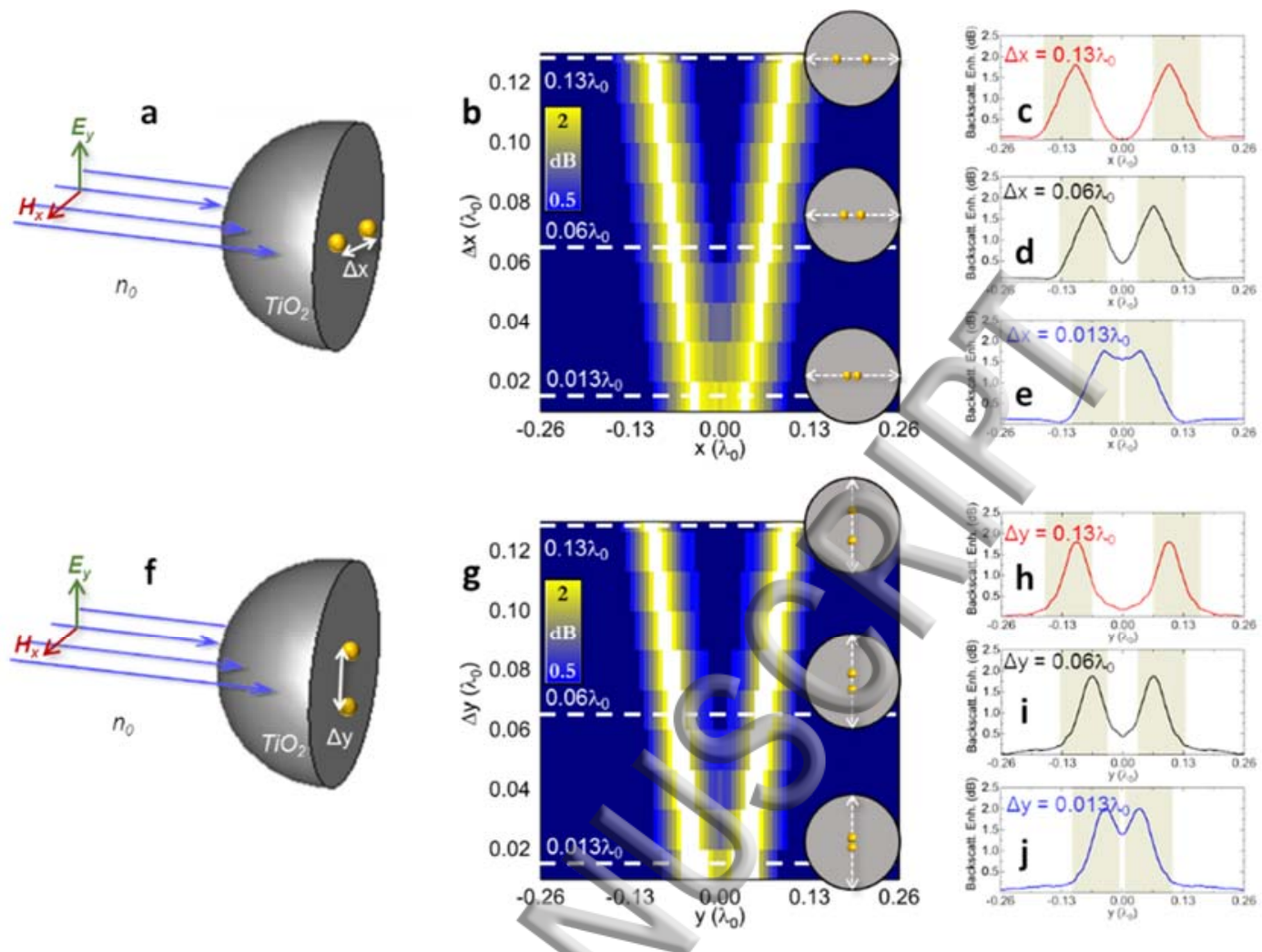

FIG.4 $(\mathrm{a}, \mathrm{f})$ Schematic representation of the setup used to evaluate the backscattering enhancement capabilities of the highindex truncated sphere. Numerical results of the backscattering enhancement distribution when two small gold spheres with diameter of $0.067 \lambda_{0}$ are placed at the output surface of the high-index truncated sphere and moved along the (b) $x$ - and (g) $y$ axis, respectively. The distance between the gold spheres is changed with the parameter $\Delta x$ and $\Delta y$ in each case respectively. Numerical results of the backscattering enhancement considering a distance between the gold spheres of (c) $\Delta x=0.13 \lambda_{0}$; (d), $\Delta x=0.06 \lambda_{0} ;$ (e) $\Delta x=0.013 \lambda_{0}$; (h) $\Delta y=0.13 \lambda_{0}$; (i), $\Delta y=0.06 \lambda_{0}$; (j) $\Delta y=0.013 \lambda_{0}$; when the Au spheres are lying and moved along the $x$ and $y$ axis, (c-e) and (h-j) respectively. Panels (c-e) and (h-j) are extracted from the white dashed lines shown in panels (b) and (f), respectively.

In the previous study, the distance between the two scatterers was changed along the $x$ or $y$ axis and they were shifted in a single axis. However, it is also interesting to evaluate the imaging capabilities of the PNJ on the whole $x-y$ plane. To evaluate it, the $x-y$ plane is scanned with the scatterers from $-0.267 \lambda_{0}$ to $0.267 \lambda_{0}$ with a step of $0.0067 \lambda_{0}$ in both $x$ and $y$ directions (see insets of Fig. 5 for a schematic representation), with the metallic spheres aligned parallel to the $x$ axis and considering two distances between them: $\Delta x=0.013 \lambda_{0}$ and $\Delta x=0.06 \lambda_{0}$. The numerical results of the backscattering enhancement in the cases studied are shown in Fig. 5a,b, respectively. In the maps obtained, an image of the metallic spheres on the $x-y$ plane is clearly observed in the central region. When $\Delta x=0.013 \lambda_{0}$ (Fig. 5a) the resulting image is slightly blurred and the subwavelength gap between the two spheres is not fully detectable, in agreement with the results discussed in Fig. 4 . However, when $\Delta x=0.06 \lambda_{\rho}$, a clearer image is achieved and the gap between the metallic spheres is correctly resolved, demonstrating the imaging capabilities of the PNJ produced by truncated high-index sphere. 

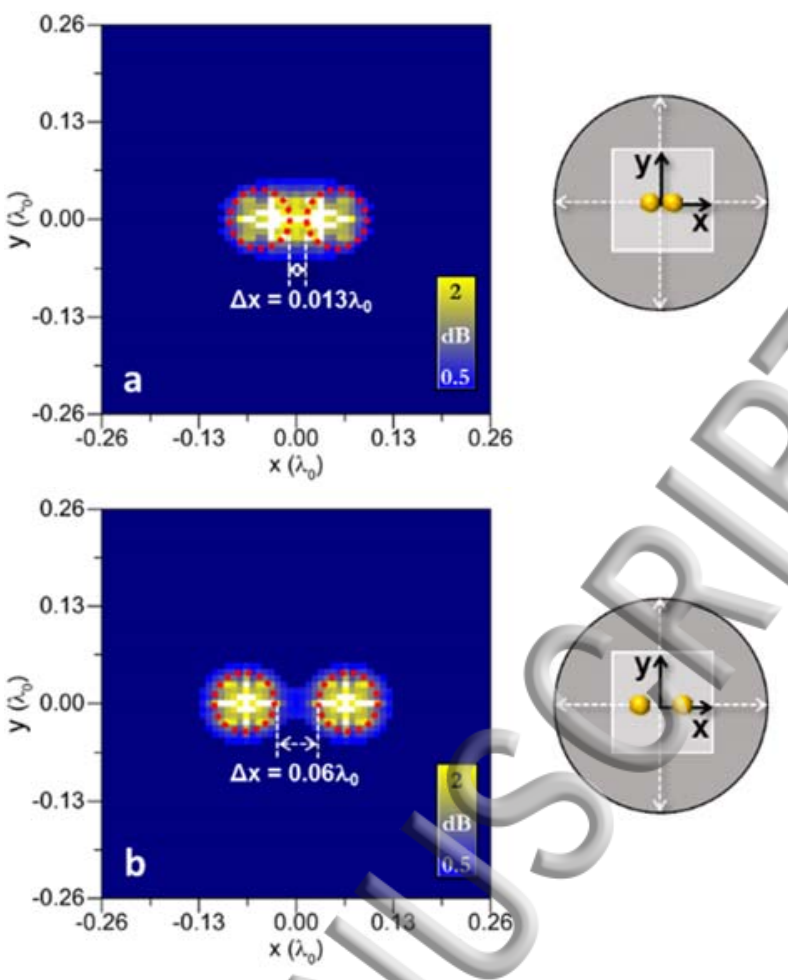

FIG.5 Numerical results of the backscattering enhancement produced when two Au spheres with diameter $0.067 \lambda_{0}$ are placed at the output surface of the truncated high-index sphere separated by a distance (a) $\Delta x=0.013 \lambda_{0}$ or (b) $\Delta x=0.06 \lambda_{0}$ and moved on the $x-y$ plane from $-0.267 \lambda_{0}$ to $0.267 \lambda_{0}$ with a step of $0.0067 \lambda_{0}$ in both $x$ and $y$ directions. A schematic representation of the setup used to obtain the image of the Au spheres is shown in the right-hand side of each panel. The grey circle and white square represent the truncated sphere (front view) and the region where the metallic spheres were shifted (are shown in the left panels), respectively.

\section{CONCLUSIONS}

To sum up, truncated high-index dielectric cylinders (2D) and spheres (3D) have been proposed as a devices to produce PNJs. The devised structures, immersed in air, have been numerically evaluated demonstrating that a focal spot is produced just at output surface with an extremely high spatial resolution of $\sim 0.14 \lambda_{0}$ and $\sim 0.06 \lambda_{0}$, for the cylinder and the sphere, respectively, improving largely the typical resolution achieved using conventional dielectrics. Furthermore, thanks to the use of a high-index material, mesoscale particles can be employed, in contrast to the large size of classical particles used to generate PNJs. The imaging capabilities of the truncated sphere have been evaluated by using the generated PNJ as a variation of the scanning-probe microscopy technique. This study has been analyzed in terms of the backscattering enhancement produced when two metallic spheres are placed near the PNJ region and moved along the transversal $x$ and $y$ axes demonstrating that the image of subwavelength features as small as $0.06 \lambda_{0}$ can be successfully detected. The present mechanism could also be applied to other geometrical structures such as cubes and ellipses, which are now under study. 
This manuscript was accepted by J. Appl. Phys. Click here to see the version of record.

\section{ACKNOWLEDGEMENTS}

V.P.-P. is supported by the Newcastle University (Newcastle University Research Fellow). M. B. acknowledges support by the Spanish Ministerio de Economía y Competitividad under contract TEC2014-51902-C2-2-R.

\section{REFERENCES}

${ }^{1}$ M. Born and E. Wolf, Principles Of Optics, 7th ed. (Cambridge University Press, New York, 1999).

${ }^{2}$ S.M. Mansfield and G.S. Kino, Appl. Phys. Lett. 57, 2615 (1990).

${ }^{3}$ K.A. Serrels, E. Ramsay, P.A. Dalgarno, B.D. Gerardot, J.A. O'Connor, R.H. Hadfield, R.J. Warburton, and D.T. Reid, J. Nanophotonics 2, 021854 (2008).

${ }^{4}$ W. Fan, B. Yan, Z. Wang, and L. Wu, Sci. Adv. 2, 40 (2016).

${ }^{5}$ I. V. Minin and O. V. Minin, Chinese Opt. Lett. 12, 060014 (2014).

${ }^{6}$ A. V. Maslov and V.N. Astratov, Appl. Phys. Lett. 108, 051104 (2016).

${ }^{7}$ Z. Wang, W. Guo, L. Li, B. Luk'yanchuk, A. Khan, Z. Liu, Z. Chen, and M. Hong, Nat. Commun. 2, 218 (2011).

${ }^{8}$ H. Yang, R. Trouillon, G. Huszka, and M.A.M. Gijs, Nano Lett. 16, 4862 (2016).

${ }^{9}$ A.M.H. Wong and G. V Eleftheriades, Sci. Rep. 5, 8449 (2015).

${ }^{10}$ K. Huang, H. Ye, J. Teng, S.P. Yeo, B. Luk'yanchuk, and C.-W. Qiu, Laser Photon. Rev. 8, 152 (2014).

${ }^{11}$ S. Legaria, V. Pacheco-Peña, and M. Beruete, Photonics 5, 56 (2018).

12 J.B. Pendry, Phys. Rev. Lett. 85, 3966 (2000).

${ }^{13}$ N. Fang, H. Lee, C. Sun, and X. Zhang, Science 308, 534 (2005).

${ }^{14}$ V. Pacheco-Peña, N. Engheta, S. Kuznetsov, A. Gentselev, and M. Beruete, Phys. Rev. Appl. 8, 034036 (2017).

15 T. Roy, E.T.F. Rogers, and N.I. Zheludev, Opt. Express 21, 7577 (2013).

${ }^{16}$ Z. Chen, A. Taflove, and V. Backman, Opt. Express 12, 1214 (2004).

${ }^{17}$ Z. Chen, A. Taflove, and V. Backman, Opt. Lett. 31, 389 (2006).

18 A. Heifetz, S.-C. Kong, A. V Sahakian, A. Taflove, and V. Backman, J. Comput. Theor. Nanosci. 6, 1979 (2009).

${ }^{19}$ X. Li, Z. Chen, A. Taflove, and V. Backman, Opt. Express 13, 526 (2005).

${ }^{20}$ B. Born, J.D. Krupa, S. Geoffroy-Gagnon, and J.F. Holzman, Nat Commun 6, 8097 (2015).

${ }^{21}$ L. Zhao and C.K. Ong, J. Appl. Phys. 105, 123512 (2009).

${ }^{22}$ B.S. Luk'yanchuk, R. Paniagua-Domínguez, I. Minin, O. Minin, and Z. Wang, Opt. Mater. Express 7, 1820 (2017).

${ }^{23}$ L. Yue, B. Yan, J.N. Monks, R. Dhama, Z. Wang, O. V. Minin, and I. V. Minin, Ann. Phys. 530, 1800032 (2018).

${ }^{24}$ S. Lecler, Y. Takakura, and P. Meyrueis, Opt. Lett. 30, 2641 (2005).

${ }^{25}$ A. Devilez, B. Stout, N. Bonod, and E. Popov, Opt. Express 16, 14200 (2008).

${ }^{26}$ D. Grojo, N. Sandeau, L. Boarino, C. Constantinescu, N. De Leo, M. Laus, and K. Sparnacci, Opt. Lett. 39, 3989 (2014).

${ }^{27}$ L. Cheng-Yang and H. Kai-Lung, Opt. Lett. 40, 5303 (2015).

${ }^{28}$ C.Y. Liu and F.C. Lin, Opt. Commun. 380, 287 (2016).

${ }^{29}$ Z. Hengyu, C. Zaichun, C.T. Chong, and H. Minghui, Opt. Express 23, 6626 (2015).

${ }^{30}$ V. Pacheco-Peña, M. Beruete, I. V. Minin, and O. V. Minin, Appl. Phys. Lett. 105, 084102 (2014).

${ }^{31}$ V. Pacheco-Peña, M. Beruete, I. Y Minin, and O. V Minin, Opt. Lett. 40, 245 (2015).

${ }^{32}$ H.H. Nguyen Pham, S. Hisatake, O.V. Minin, T. Nagatsuma, and I.V. Minin, APL Photonics 2, 056106 (2017).

${ }^{33}$ Y.E. Geints, A.A. Zemlyanov, and E.K. Panina, J. Opt. Soc. Am. B 28, 1825 (2011).

${ }^{34}$ V.N. Astratov, J.P. Franchak, and S.P. Ashili, Appl. Phys. Lett. 85, 5508 (2004).

${ }^{35}$ I. V. Minin, O. V. Minin, V. Pacheco-Peña, and M. Beruete, Appl. Phys. Lett. 106, 1 (2015).

${ }^{36}$ V. Pacheco-Peña, I. V. Minin, O. V. Minin, and M. Beruete, SPIE Newsroom 102, 171109 (2016).

${ }^{37}$ M. Gerlach, Y.P. Rakovich, and J.F. Donegan, Opt. Express 15, 17343 (2007).

${ }^{38}$ D. Ju, H. Pei, Y.Jiang, and X. Sun, Appl. Phys. Lett. 102, 171109 (2013).

${ }^{39}$ V. Pacheco-Peña, I. V Minin, O. V Minin, and M. Beruete, Ann. Phys. 528, 684 (2016).

${ }^{40}$ I. V. Minin, O. V. Minin, V. Pacheco-Peña, and M. Beruete, Quantum Electron. 46, 555 (2016).

${ }^{41}$ A. Kovrov, A. Novitsky, A. Karabchevsky, and A.S. Shalin, Ann. Phys. 530, 1800129 (2018).

${ }^{42}$ V. Pacheco-Peña, N.A. Haritoshin, I. V Minin, O. V Minin, and M. Beruete, in 9th Int. Congr. Adv. Electromagn. Mater. Microwaves Opt. (METAMATERIALS), 2015 (2015), pp. 463-465.

${ }^{43}$ V. Pacheco-Pena, M. Beruete, M. Navarro-Cia, I. V. Minin, and O. V. Minin, in 2015 Usn. Radio Sci. Meet. (Joint with APS Symp. (IEEE, 2015), pp. 200-200.

${ }^{44}$ I. V Minin, O. V Minin, V. Pacheco-Peña, and M. Beruete, Opt. Lett. 40, 2329 (2015).

${ }^{45}$ V.R. Dantham, P.B. Bisht, and C.K.R. Namboodiri, J. Appl. Phys. 109, 103103 (2011).

${ }^{46}$ I.V. Minin, O. V. Minin, and I.S. Nefedov, Opt. Lett. 41, 785 (2016).

${ }^{47}$ R. Yahiaoui, P. Mounaix, V. Vigneras, U.-C. Ching Seu, C. Elissalde, and M. Maglione, in 43rd Eur. Microw. Conf. (European Microwave Association, 2013), pp. 1159-1162. 
Publishing ${ }^{48}$ P. Ferrand, J. Wenger, A. Devilez, M. Pianta, B. Stout, N. Bonod, E. Popov, and H. Rigneault, Opt. Express 16, 6930 (2008). 49 E. Cyp and M. Sprague, Nature 398, 761 (1999).

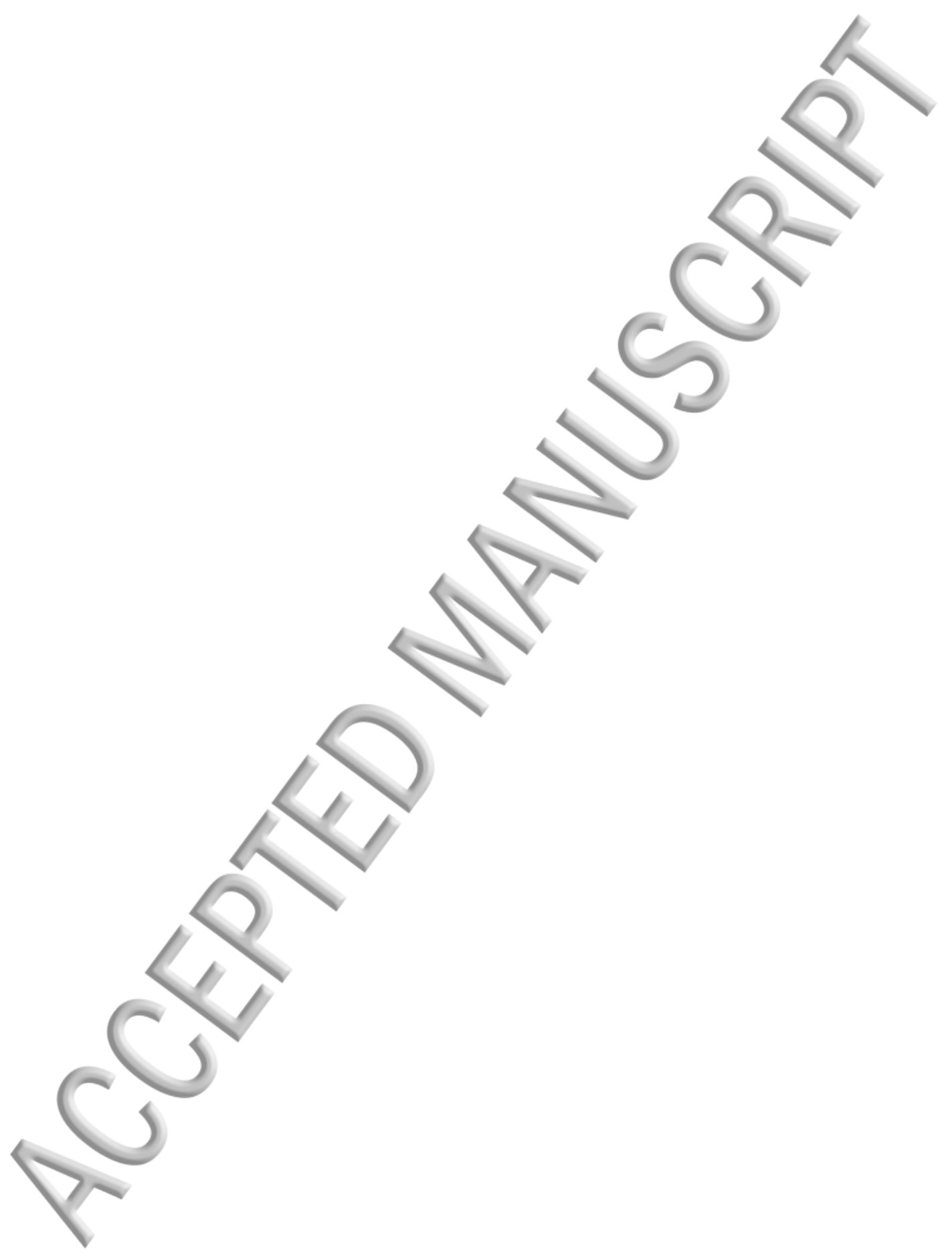



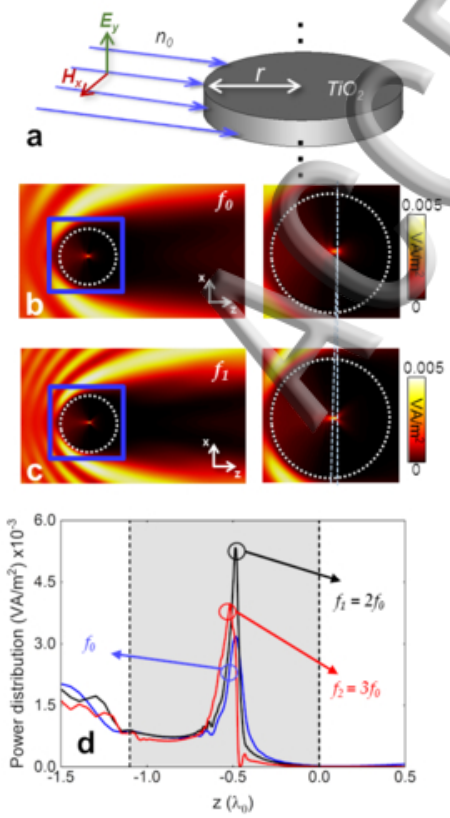

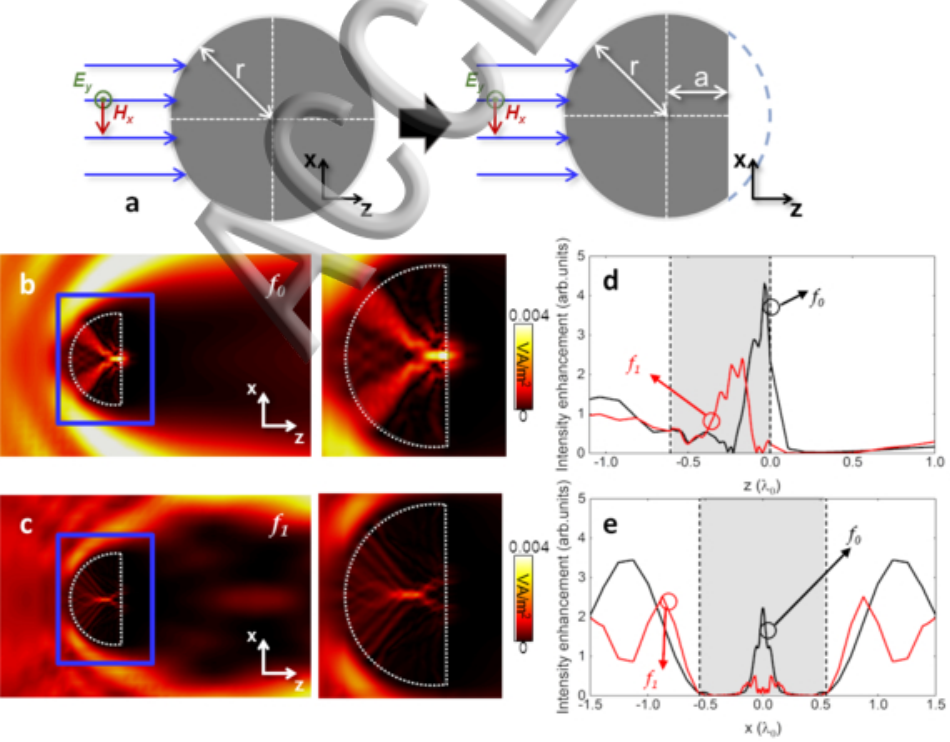

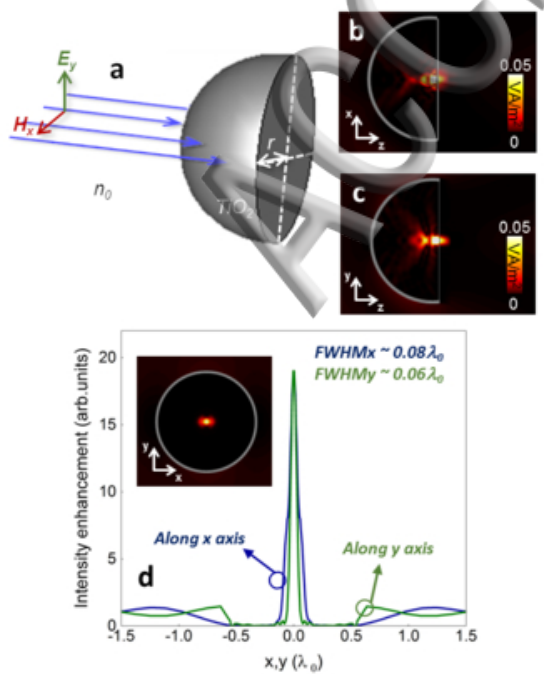

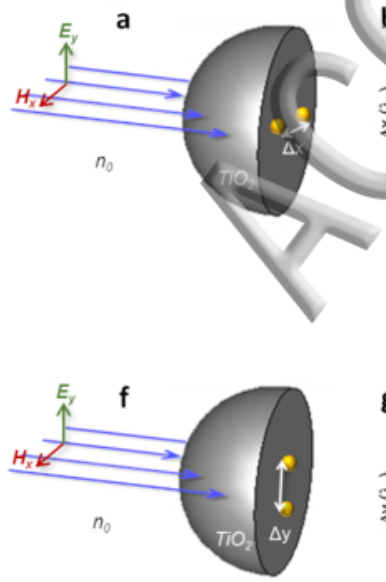

b

\section{$0.120 .13 \pi_{0}$}

30.080 .5

× $\quad 0.06 \lambda_{0}$

0.06

0.04

$0.020 .013 \lambda_{0}$

$-0.26$

$-0.13$

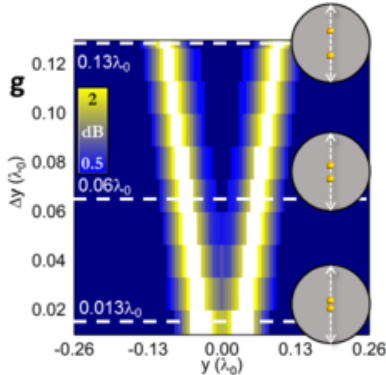

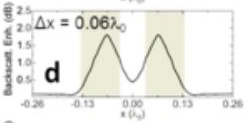
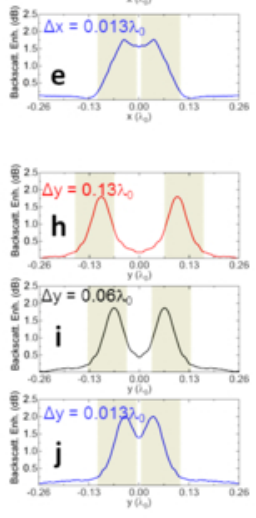

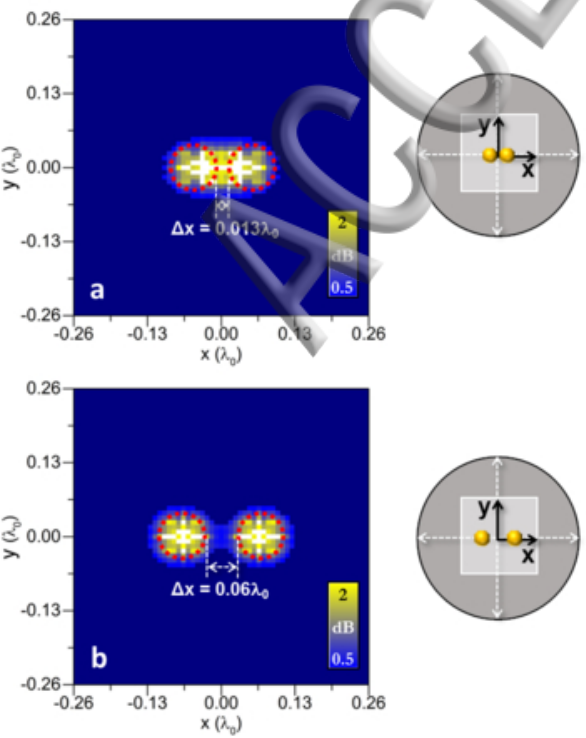